Introduction The pathogenesis of Inflammatory Bowel Disease (IBD) is unclear which hinders effective targeted drug development. IBD and murine models of colitis are associated with the abnormal accumulation of activated dendritic cells (DCs) in the colonic epithelium. DCs play a critical role in promoting inflammatory responses and blockade of their activation prevents colitis development in mouse models. We now propose to address the mechanisms underlying the aberrant accumulation of DCs in the gut by focusing on microbial danger stimuli that drive activation and migration of DCs. Methods We analysed the expression of migration-associated markers on DCs from normal and colitic mice. Bone marrow derived DCs (BMDCs) from WT or Beta-2 Integrin ${ }^{-/}\left(\right.$ITGB2 $\left.^{-/}\right)$mice were cultured in vitro and their migration and activation analysed in response to control (Phosphate buffered saline - PBS), bacterial lipopolysaccharide (LPS), live Escherichia coli (EC), and live Bacteroides fragilis $(\mathrm{BF})$ in the presence and absence of the lamina propria extracellular matrix component fibronectin.

Results During colitis there was a marked increase in a population of CD103- ( $a_{\mathrm{E}}$ integrin) DCs. We were able to mirror these populations in vitro. DCs moved via random motion and their velocity after stimulation with LPS and EC, in the absence of fibronectin, was significantly decreased. In comparison, stimulation with BF significantly increased DC velocity ( $p<0.001$ for all). In the presence of fibronectin, there was no change in DC velocity. The track displacement length (the distance between the start and finishing point of a given cell migration track) was significantly decreased after EC stimulation and significantly increased after BF stimulation ( $p<0.01$ for both). Unstimulated ITGB2 $\%$ BMDC velocity and track displacement length were significantly increased in comparison to that of unstimulated WT BMDC ( $p<0.001$ for both). This was more marked in the absence of fibronectin.

Conclusion We have shown that in vitro WT DC cultures contain DCs with similar integrin-defined phenotypes to those found in colonic DCs in colitis. Differential bacterial stimulation causes opposing fibronectin-dependent effects on BMDC migratory behaviour whilst absence of ITGB2 significantly alters the migratory behaviour of BMDC. Our data implicates a complex relationship between specific components of gut microbiota, extracellular matrix, and migration and activation of DCs that could potentiate the aberrant accumulation of DCs in the colitic gut. If this complex relationship is further elucidated, it may be possible in the future to develop therapies that reduce colitis by controlling DC migration.

Disclosure of Interest None Declared.

\section{PTH-104 THE ROUTINE MEASUREMENT OF THIOPURINE METABOLITE LEVELS RESULTS IN DOSE OPTIMISATION IN ONE THIRD OF IBD PATIENTS; RESULTS FROM A DISTRICT GENERAL HOSPITAL}

doi:10.1136/gutjnl-2013-304907.591

${ }^{1} \mathrm{H}$ M Dewhurst, ${ }^{2} \mathrm{H}$ E Johnson, ${ }^{3} \mathrm{~J}$ Begley, ${ }^{2} \mathrm{~S}$ A Weaver, ${ }^{2,}$ S D McLaughlin. ${ }^{1}$ Research; ${ }^{2}$ Gastroenterology; ${ }^{3}$ Clinical Biochemistry, Royal Bournemouth Hospital, Bournemouth, UK

Introduction Measuring azathioprine or mercaptopurine (AZA) metabolite levels 6-TGN and 6-MMPN allows identification of patients who are: 1 . Non compliant with their medication, 2. On a sub-optimal doe, 3. On a supra-therapeutic dose, 4. Are preferentially metabolising azathioprine to methylated metabolites (6-MMPN:6-TGN ratio > 11).

Our own and others published data demonstrate that measuring metabolite levels in patients failing azathioprine therapy followed by appropriate changes in dosing and/or the addition of allopurinol (with $75 \%$ dose reduction in AZA) can result in clinical remission in the majority of patients 1 . We report the outcome of the routine measurement of metabolite levels in patients treated with AZA who were in a clinical remission without side effects or abnormal liver function tests (LFTs).

Methods All patients underwent TPMT testing, azathioprine and mercaptopurine were initiated at doses of $2 \mathrm{mg} / \mathrm{kg}$ and $1 \mathrm{mg} / \mathrm{kg}$ respectively in those with wild-type TPMT with a $50 \%$ reduction in dose in TPMT heterozygotes. We searched the prospective database maintained by our biochemistry department for all patients who underwent metabolite level testing from September 2011 to November 2012, hospital case notes for these patients were reviewed. The indications, results of testing, changes in clinical management and patient outcomes were recorded.

Results 108 patients underwent metabolite testing, median length of follow-up since testing was 287.6 days (range 21-441), all were stable on AZA for $>4$ weeks with normal LFTs and in a clinical remission. 38 (35.2\%) had UC, 66 (61.1\%) CD, 52 (48.1\%) were male.

$17(15.7 \%)$ patients had a sub-theraputic 6-TGN, 10 (9.3\%) supra-theraputic 6-TGN level $(>800)$ all of whom had dose optimisation. $6(5.6 \%)$ patients were hypermethylators these were switched to allopurinol co-therapy with an appropriate reduction in AZA dose.

Conclusion In the present study the routine measurement of AZA metabolites resulted in a change in clinical management in $30.6 \%$ of patients.

Whilst unproven in prospective longitudinal studies logic suggests that the routine measurement of AZA metabolites in all patients commenced on thiopurines followed by appropriate dose optimisation (with or without allopurinol co-prescription) should reduce or prevent the development of drug side effects, abnormal LFTs and bone marrow suppression and reduce the risk of disease relapse. We recommend that AZA metabolite testing is performed in all patients 4-6 weeks after commencing AZA.

Disclosure of Interest None Declared.

\section{PTH-105 THE EFFECT OF INFLIXIMAB PRE-TREATED HUMAN BLOOD- ENRICHED DENDRITIC CELLS FROM PATIENTS WITH ACTIVE CROHN'S DISEASE AND HEALTHY CONTROLS ON SUBSEOUENT HUMAN T-LYMPHOCYTE PHENOTYPE AND CYTOKINE PRODUCTION IN VITRO}

doi:10.1136/gutjnl-2013-304907.592

1. S T C Peake, ${ }^{2} \mathrm{D}$ Bernardo, ${ }^{2} \mathrm{E}$ Mann, ${ }^{1} \mathrm{~J}$ Landy, ${ }^{2} \mathrm{H}$ Omar, ${ }^{2} \mathrm{~S}$ C Knight, ${ }^{1} \mathrm{~A}$ L Hart. ${ }^{1} / B D$ Unit, St Mark's Hospital; ${ }^{2} A P R G$, Imperial College, London, UK

Introduction Dendritic cells (DC) play a key role in discriminating between commensal microorganisms and potentially harmful pathogens. Expression of surface markers and cytokine production by DC at the time of antigen presentation control T-cell differentiation, cytokine profile \& homing properties imprinted on stimulated T-cells. This process defines the type of immune response that occurs and its anatomical location. In $\mathrm{CD}$, dysregulation of the immune response to gut microbiota and aberrant immune cell trafficking play a central role in disease pathogenesis. Infliximab (IFX) is an effective treatment for CD, but its mechanism of action is unclear. In this study, we investigated the effect of IFX pre-treated blood-enriched DC, isolated from patients with active CD and healthy controls (HC), on human T-cell proliferation, phenotype\&cytokine production

Methods Low density cells (LDC), enriched for DC, were obtained following Ficoll and Nycoprep gradient separation of fresh blood from patients with active ileocolonic CD (CDAI > 220) and HC. LDC were cultured $\left(0.5 \times 10^{6} \mathrm{cells} / \mathrm{ml}\right)$ with IFX $(1.10 \mu \mathrm{g} \& 100 \mu \mathrm{g} /$ ml\&basal) for $24 \mathrm{hr}$. T-cells were enriched from allogeneic HC blood and labelled with CFSE. LDC were added to T-cells in complete medium $(400,000$ cells $/ \mathrm{ml})$ at basal,1.2\&3\% concentrations and incubated for 5 days. Following incubation, T-cell proliferation, 
expression of $\beta 7 \& C L A$ surface homing markers and cytokine content of T-cells (TNF $\alpha$, TGF $\beta$, IFN $\gamma$, IL-10.15.17) was quantified by flow cytometry on stimulated T-cells. Unparied t-test and one-way ANOVA statistical analyses were applied

Results In basal conditions, LDC from HC \& CD patients did not differ in their stimulatory capacity for allogeneic T-cells or in the cytokine profile acquired by T-cells. However, T-cells stimulated by $\mathrm{LDC}$ from $\mathrm{CD}$ patients decreased $\beta 7$ intensity ratio. Following culture with IFX, LDC decreased their stimulatory capacity in a dose-dependent, stepwise fashion in both HC and CD. Culture with IFX did not have any effect on the acquired homing profile of stimulated T-cells, although these T-cells had a trend (not statistically significant) towards lower TNF $\alpha$ and higher IL-17 production

Conclusion The marked reduction in the ability of LDC to stimulate T-cells following culture with IFX represents one plausible explanation for the efficacy of anti TNF-alpha therapies in the treatment of CD. This effect was dose-dependent (within our range of test concentrations) suggesting that higher doses of IFX further reduce T-cell stimulation and may provide one explanation of the clinical benefits of dose escalation in refractory CD

Disclosure of Interest None Declared.

\section{PTH-106 UNRAVELLING THE MECHANISM OF ACTION OF INFLIXIMAB IN CROHN'S DISEASE AND HEALTHY CONTROLS FOLLOWING IN VITRO CULTURE WITH BLOOD- ENRICHED DENDRITIC CELLS}

doi:10.1136/gutjnl-2013-304907.593

${ }^{1, *} \mathrm{~S}$ T C Peake, ${ }^{2} \mathrm{D}$ Bernardo, ${ }^{2} \mathrm{E}$ Mann, ${ }^{1} \mathrm{~J}$ Landy, ${ }^{2} \mathrm{H}$ Omar, ${ }^{2} \mathrm{~S}$ C Knight, ${ }^{1} \mathrm{~A}$ L Hart. ${ }^{1} / \mathrm{BD}$ Unit, St Mark's Hospital; ${ }^{2} A P R G$, Imperial College, London, UK

Introduction Dendritic cells (DC) play a key role in discriminating between commensal microorganisms and harmful pathogens. DC phenotype and cytokine production determine the type of immune response elicited by T-cells following antigen presentation. DC also direct the T-cells to target tissues to perform their function via imprinting tissue-specific homing markers. In $C D$, dysregulation of the immune response to gut microbiota \& aberrant immune cell trafficking play a role in disease pathogenesis. Infliximab (IFX) is an effective treatment for CD but its mechanism of action is unclear. In this study we investigated the in-vitro effect of IFX on phenotype \& ongoing cytokine production of human blood-enriched DC from patients with active CD and healthy controls (HC)

Methods Low density cells (LDC), enriched for DC, were obtained following Ficoll \& Nycoprep separation of blood from patients with active CD (CDAI > 220) and HC. LDC were cultured $\left(0.5 \times 10^{6}\right.$ cells/ $\mathrm{ml})$ with IFX $(1.10 \& 100 \mu \mathrm{g} / \mathrm{ml} \&$ basal $)$ for $24 \mathrm{hr}$. Activation marker (CD40, CD80, HLADR), TLR receptor (TLR2.4) and homing marker (CCR4 5.7.8.9.10, $\beta 7$ ) expression was quantified by flow cytometry. Natural ongoing intracellular cytokine production (TNF $\alpha$, TGF $\beta$ \&IL-6.10.12.15) was assessed via intracellular staining and flow cytometry. Cytokine secretion was measured on cell-free culture supernatants via Multiplex. Unpaired t-test and one-way ANOVA statistical analyses were applied

Results TNF $\alpha$ and IL- 6 were increased in culture supernatants from $\mathrm{CD}$ although their intracellular ongoing cytokine production was decreased. LDC from CD had decreased $\beta 7$ (gut-homing integrin) expression. Following IFX culture, LDC decreased $\beta 7$ expression \& CCR9 intensity ratio (dose-dependent). There was a trend towards reduction in TLR2 and 4 expression (not statistically significant). IL-12 production by LDC from HC was increased following IFX culture. There was a marked reduction in TNF $\alpha$ and IL-12 in cell supernatant following culture with IFX
Conclusion Increased TNF $\alpha$ and IL- 6 in culture supernatants from $\mathrm{CD}$ patients coupled with a decrease in ongoing production by DC suggests a negative cytokine feedback system. Reduced $\beta 7$ expression on LDC from CD patients may suggest that DC have already been recruited to the site of mucosal inflammation. Further experiments are needed to confirm this. Reduced expression of CCR9 and elevated production of IL-12 in LDC cultured with IFX, shows reduced affinity for gut-homing and increased immunogenicity and may suggest a possible mechanism for IFX-induced paradoxical inflammation. The most dramatic effect of IFX was a reduction in TNF $\alpha$ in the supernatant, which is likely to represent neutralisation of the cytokine

Disclosure of Interest None Declared.

\section{PTH-107 KNOWLEDGE OF SOUTH ASIAN POPULATION IN INFLAMMATORY BOWEL DISEASE: CCKNOW- HOW MUCH DO THEY KNOW?}

doi:10.1136/gutjnl-2013-304907.594

1.'S Soteriadou, 'S Hickman, 'J K Limdi. 'Gastroenterology, Pennine Acute NHS Trust, Manchester, UK

Introduction Despite an unprecedented explosion in information and technology and improved understanding of the aetiopathogenesis of Inflammatory bowel diseases (IBD) it remains unclear whether this has translated into a meaningful improvement in patients understanding of their disease. Less is known about South Asian patients with IBD. Our aim was to assess the understanding amongst South Asian patient's using the validated CCKNOW questionnaire ${ }^{1}$.

Methods We conducted a prospective questionnaire-based study of 108 South Asian IBD patients at our institution. Patient demographics, first language, country of origin, educational level and disease duration were recorded. A previously validated 24-item Crohn's and Colitis Knowledge (CCKNOW) questionnaire was used to assess IBD-specific knowledge relating to disease ${ }^{1}$.

Results Forty-three of 108 surveys sent out were returned (39.8\%).

Twenty-three of the 43 patients surveyed were male (53.5\%). Fifteen were born in the UK (34.8\%). English was recorded as the first language in 15 (34.9\%). The highest education level was high school comprehensive in 19 (44.1\%), sixth-form/technical college in 5 $(11.6 \%)$, university in $14(32.6 \%)$, and postgraduate in $3(6.98 \%)$. Fifty one percent of patients described their health to be average or below average, as opposed to good.

Both the mean and median CCKNOW score were 6.74 (Range $0-20)$. Mean scores related to anatomy were 1.21 ( $\mathrm{n}=5 ; 24.1 \%)$, aetiology $0.77(n=2 ; 38.35 \%)$, symptoms $1.55(n=3 ; 38.7 \%)$, diagnosis $0.19(\mathrm{n}=1 ; 18.6 \%)$, treatment and related complications 1.91 $(\mathrm{n}=7 ; 23.6 \%)$, surgery $0.07(\mathrm{n}=1,7 \%)$, complications $0.93(\mathrm{n}=4$; $23.2 \%)$, and pregnancy and fertility related questions $0.12(\mathrm{n}=1$; $11.6 \%)$

Conclusion The knowledge base of South Asian IBD patients was thus deemed poor. More concerning were specific areas of deficient knowledge such as pregnancy and fertility, cancer and treatment related complications with the potential for serious consequences.

Patient education is a key determinant of clinician -patient relationship and an important outcome modifier in chronic disease. IBD teams must guide information needs of specific patient groups through well designed material to enhance knowledge and understanding of disease ultimately impacting on quality of life, treatment adherence and reduction in the use of healthcare resources

Disclosure of Interest None Declared.

\section{REFERENCE}

1. Eaden JA, Abrams K, Mayberry JF. The Crohn's and Colitis Knowledge Score: a test for measuring patient knowledge in inflammatory bowel disease. Am J Gastroenterol. 1999 Dec; 94(12):3560-6. 